\title{
POINTING AND VISUALIZATION
}

\author{
William C. Hill and James D. Hollan
}

\author{
Computer Graphics and Interactive Media Research Group \\ Bellcore, 445 South Street, Morristown, NJ 07962-1910
}

Email: willhill@bellcore.com, hollan@bellcore.com

\begin{abstract}
The nature of visualizations and the social uses to which they are put rely heavily on pointing behavior. In the context of a switched telephone network visualization, this tape illustrates novel task-specific pointing facilities.
\end{abstract}

Keywords: Pointing, visualization, graphical user interface, visual attention, interface mechanisms.

\section{INTRODUCTION}

The accepted wisdom of what tools and skills are required to produce visualization excellence is undergoing redefinition in order to incorporate, among other changes, new pointing facilities. These facilities are enabled for the first time by the controlled dynamics that computational graphics offer. The facilities wbecame visibleill be in demand because they will allow users to express complex task-specific pointing intentions.

Broadly construed, pointing involves directing visual attention. Pointing with your index finger is a simple example. Just turning your head to look may point if it directs the visual attention of others. Using a laser-pointer during a presentation is another common example. But so is explaining travel directions with maps over the phone by verbally directing the visual attention of another to specific map locations. Theatrical lighting is a more complex form of pointing. For example, the synchronous dimming of one spotlight and brightening of another serves to direct audience attention. So too, the many and varied ways film directors direct visual attention around movie scenes serve as sophisticated examples. In the realm of human computer interfaces pointing is almost synonymous with using the mouse. Normally the mouse is used to direct the flow of control but during demonstration users employ the cursor to direct others' visual attention. As should be clear from these examples, pointing mechanisms vary widely and are often central to effective communication.

Rarely are graphics employed for strictly private individual purposes. More commonly, through meetings, presentations, publications, and mass media, graphics are employed as social instruments, shared among individuals and groups. Social use of graphics is the norm. Empirical observation shows that the social use of graphics relies heavily on pointing behaviors, often in coordination with speech or text. The paradigmatic example is a presenter pointing at presentation graphics before a live audience but there are many other social situations where graphics serve as shared interactionorganizing artifacts. Visualizations are such social graphics and as expected, if you just look, the amount of pointing by hand, mouse and cursor, narrative reference, and embedded visual technique during their use is enormous. Thus, from an empirical point of view, pointing is fundamental to visualization.

For understanding the existing practice of pointing as the ground upon which to build more effective pointing facilities, three observations are paramount. First, pointing behaviors commonly realize intents more complex than look here. By analyzing pointing performances captured on videotape we have produced a preliminary taxonomy of such complex pointing intents. Because pointing behaviors realize complex intents, the simplistic assertion that a mouse and cursor handle the majority of interactive pointing needs is suspect. Second, graphical resources for pointing differ according to medium and situation. This amounts to saying we should expect and prepare for task-specific pointing techniques. Third, by reifying pointing behaviors and bringing them into the graphics themselves, computational techniques radically alter what pointing can be. 


\section{TASK-SPECIFIC POINTING IN VISUALIZATION}

In the realm of visualization, the narrative use of graphics almost always involves pointing. Good visualizations, for example, are rich with the interplay of beautiful and subtle pointing. Choices of lighting, materials, highlights, textures, point of view, size, transparency, cutouts, arrows, motion blur, eye-catching movement, time dilation, edge-pushing, burnout, to name only a few, are mechanisms employed to direct visual attention. Fast rendering speeds, double-buffering, 3D geometry, and texture mapping techniques now common to current visualization efforts extend the power of graphic transformation and provide new ways for expressing pointing intentions that employ the preattentive powers of human vision. Until recently, these methods have been unavailable.

But not every use of graphic technique is pointing. Otherwise pointing would mean nothing more than graphic technique and that is not the case. Although most graphic techniques may be employed to point, in any given image only a few serve pointing intent. Only if you can remove the pointing and still have the image do you have pointing. The kind of pointing we are discussing is separable, removable, and temporary. Thus, in practice, on separate occasions, a presenter, having conceived particular communicative purposes relevant to distinct audiences, will point at the same image in different ways tailored to those purposes.

Our example concerns the visualization of switched telephone network activity, in particular the performance of digital signals on fiber optic cables connecting mobile telephone transceiver sites to a central telephone switching office. These digital signals run at $45 \mathrm{Mb} / \mathrm{second}$ but are subject to transient errors of various types. Special monitoring hardware collects error data so that performance can be examined to attempt to find predictive patterns. However, depending on the number of signals, number of error parameters per signal, and the frequency of collection one frequently confronts an overwhelming volume of data. One such collection effort with which we are involved collects over 100,000 error parameters per day.

We have built a visualization of these data for digital signal experts. They are interested in improving the quality of digital service so that one might be able to guarantee that transient errors will not occur more often than once in, say, $10^{-8}$ clock cycles or some other suitably low level. The users of our visualization are concerned, among other things, with seeing error threshold breaking in the context of previous patterns of errors. That is to say, they want to see what is going on with the errors in periods preceding that in which particular thresholds are broken.
Their goal is to detect error signatures predictire of future threshold breaking. In terms of visualization, this means we wanted to direct their attention at the occurrince of threshold-breaking events but in a way that also highlights potentially relevant subthreshold error data. The visualization technique we developed serves as an example of task-specific pointing. We refer to the pointing intention as threshold-breaking in context.

The threshold-breaking in context pointing intention is served by a variably-transparent movable neutral grey cutting plane to represent the current threshold as set by the user. The transparent grey plane creates a contrast luminance between errors that exceed the threshold and those under the threshold. Within the dynamic scene, this creates a popout effect [1] in which the eye is effortlessly drawn specifically to the threshold-breaking errors. Transparency handles the other part of the pointing intention --- not to occlude error activity under the threshold. Subthreshold activity can still be discerned and users can manipulate a transparency control to get more or less visual stimulation from subthreshold activity. Users of this prototype system have found this technique effective. Essentially, the technique enables users to state a myriad of threshold-related questions graphically.

The intent of our video is to draw attention to the importance of pointing, construed broadly, but implemented narrowly to substitute easy visual tasks for otherwise difficult ones as they arise naturally in a real task. We think this kind of task-specific pointing will play an increasing role in future graphical interfaces and in the wider use of dynamic graphics. Techniques for exploiting computational graphics to express complex pointing intentions are just beginning to be explored. How to effectively use them is challenging, particularly for new types of applications. For example, computer supported cooperative work applications raise a variety of issues resulting from the existence of multiple points of view and the complex nature of shared tasks.

\section{ACKNOWLEDGMENT}

The authors would like to acknowledge the assistance of Larry Stead in the making of this video tape.

\section{REFERENCES}

1. Triesman, A. Features and Objects: The Fourteenth Bartlett Memorial Lecture. The Quarterly Journal of Experimental Psychology, 40A(2), 203-237, 1988. 